\title{
An iterative Power Allocation Alogrithm for Energy Efficiency Optimization in Massive MIMO Systems
}

\author{
Yuanyuan Ren ${ }^{1,-}$, Panpan $\mathrm{Miao}^{2}$ and Yanhui $\mathrm{Lu}^{3}$ \\ 11st1344349720@qq.com, Zhengzhou University,Zhengzhou,Henan,450001 China \\ 22nd1520588972@qq.com, Zhengzhou University,Zhengzhou,Henan,450001 China \\ 33rd ieyhlu@zzu.edu.cn, Zhengzhou University,Zhengzhou,Henan,450001 China
}

\begin{abstract}
In this paper, a transmitting power allocation strategy for users jointed together pre-coding is presented to eliminate inter users interference and improve the energy efficiency of Massive MIMO systems. The power allocation model formularized as energy efficiency optimization problem of the users ' power and circuit power consumption. W demonstrate that energy efficiency is a convex function of users' power. In order to reduce the complexity of traditiona power allocation algorithm, an iterative power allocation algorithm based on convex optimization theory is proposed t figure out the optimal value of each user power. The simulation results show that the iterative algorithm can effectivel improve the energy efficiency of Massive MIMO systems.
\end{abstract}

Keywords: Massive MIMO, energy efficiency, power assumption, convex function

Received on 22 February 2017, accepted on 09 April 2017, published on 14 April 2017

Copyright (C) 2017 Yuanyuan, Ren Author et al., licensed to EAI. This is an open access article distributed under the terms of the Creative Commons Attribution licence (http://creativecommons.org/licenses/by/3.0/), which permits unlimited use, distribution and reproduction in any medium so long as the original work is properly cited.

doi: 10.4108/eai.15-1-2018.154109

\section{Introduction}

The Internet of Things is an important part of the new generation of information technology. It can connect objects and bring convenience to people's lives. The fifth generation of mobile communication technology (5G) will make the Internet of things have more powerful communication capabilities, so that the Internet of Things is more widely used. In the $5 \mathrm{G}$ research and development, energy efficiency has aroused people's attention. Improving energy efficiency is not only conducive to reducing the interference of co-channel users, but also reducing the environmental pollution. MIMO is one of the most important technology to improve spectral efficiency [1]. Massive MIMO can significantly improve the energy efficiency and spectral efficiency by increasing the number of antennas at the base station ${ }^{[2]}$. But as the number of base station antennas increases, the loss of the RF chains will also increases ${ }^{[3]}$. Therefore, the power loss

Corresponding author. Yuanyuan,Ren,Email:1344349720@qq.com of RF chains must be taken into account in analyzing the energy efficiency of Massive MIMO systems ${ }^{[4]}$. At the same time we should pay attention that the less number of RF chains is used, the smaller capacity of the system will be ${ }^{[5]}$.

In recent years, there are many energy efficiency studies on MIMO systems [6] [7]. For example, in [6], it studied how to improve the energy efficiency of sensors in MIMO systems based on Alamouti diversity. Radio resource allocation is an effective way to improve energy efficiency [8]. In [9], the optimal energy efficiency allocation algorithm in OFDMA system was studied. In [10], the optimal energy efficiency algorithm is studied in the distributed antenna system. In [11], the energy efficiency of the uplink multiuser systems is analyzed. It is proved that the energy efficiency function is a quasiconcave function of the power matrix, and based on this analysis, an iterative power allocation algorithm is proposed.

The existing research results show that most power allocation algorithms usually assume that the base station 
allocates power to users evenly, ignoring the energy efficiency optimization problem when users allocated power unequally. Based on these researches, this paper eliminates the interference between users by decomposing the channel matrix and applying pre-coding technique. And then a new power consumption model is founded, which takes the users' power and circuit power into account. After we deduce the expression of energy efficiency, the convexity of energy efficiency is proved, based on convex optimization, an iterative power allocation algorithm is proposed to solve the energy efficiency optimization problem. The simulation results show that the iterative algorithm can effectively improve the energy efficiency of Massive MIMO system.

\section{System model}

In Massive MIMO downlink systems, we assumed that the number of base station antennas is $N$ and the receiver has $K$ single antenna users. The system model is shown in figure 1.

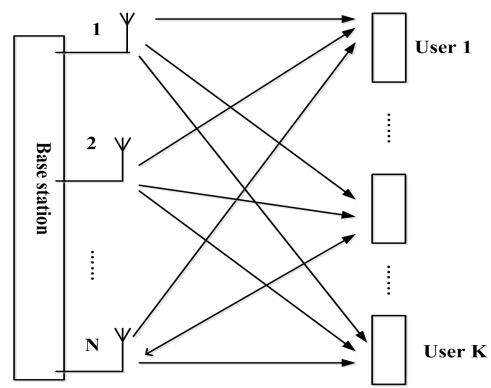

Figure 1. The model of massive MIMO system

The signal received by the client is,

$$
\mathbf{y}=\mathbf{H Q P x}+\mathbf{n} .
$$

Where $\mathbf{y}$ and $\mathbf{x}$ are the received and transmitted signal vectors of $K \times 1$ respectively ${ }^{[1]}, \mathbf{H}$ is the Rayleigh fading channel matrix of $K \times N, \mathbf{Q}$ is the pre-coding matrix of $N \times K, \quad \mathbf{n}$ is the noise vector of $K \times 1$, $\mathbf{P}=\operatorname{diag}\left\{\sqrt{p_{1}}, \sqrt{p_{2}}, \ldots, \sqrt{p_{K}}\right\}$ represents the , power allocated to each user of $K \times K$.

Using singular value decomposition (SVD),

$$
\mathbf{H}=\mathbf{U} \mathbf{\Sigma} \mathbf{V}^{H} \text {. }
$$

$\mathbf{U}$ and $\mathbf{V}$ are the unitary matrices of $K \times K$ and $N \times N$ respectively, $\boldsymbol{\Sigma}$ is the diagonal matrix of $K \times N$,

$$
\boldsymbol{\Sigma}=\operatorname{diag}\left\{\lambda_{1}, \lambda_{2}, \ldots, \lambda_{K}, 0, \ldots, 0\right\}
$$

At the receiver, we use Zero Forced $(\mathrm{ZF})$ receiver to eliminate the interference between users, the received signal can be rewritten as

$$
\begin{gathered}
\mathbf{y}^{\prime}=\mathbf{W y}=\mathbf{W H Q P x}+\mathbf{W n} . \\
\mathbf{W}=\left(\mathbf{U}^{H} \mathbf{U}\right)^{-1} \mathbf{U}^{H} .
\end{gathered}
$$

W is the linear receive of $K \times K$. Assuming that the transmitter has known the channel state information, we decomposes the modal and uses the pre-coding at the transmitter, that is $\mathbf{Q}=\mathbf{V}$. At the receiver ZF pre-coding is used. Modal decomposition diagram shown in Figure 2.

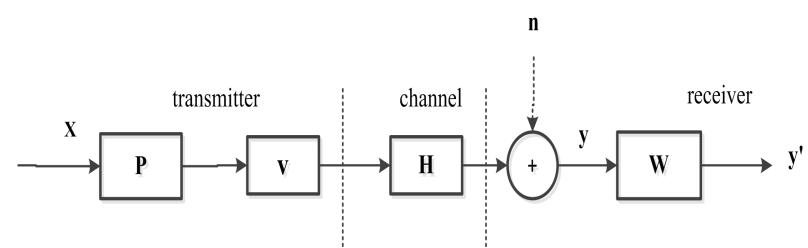

Figure 2. Modal decomposition

The received signal is reduced to,

$$
\mathbf{y}^{\prime}=\sum \mathbf{P x}+\mathbf{n}^{\prime} .
$$

Where $\mathbf{n}^{\prime}=\mathbf{W} \mathbf{n}=\left(\mathbf{U}^{H} \mathbf{U}\right)^{-1} \mathbf{U}^{H}, \mathbf{n}$ is a Gaussian white noise with a zero mean and a variance of $\sigma^{2}$. The signalto-noise ratio of the user $k$ is given by,

$$
\eta_{k}=\frac{\lambda_{k}^{2} p_{k}}{\sigma^{2}}
$$

Then the total capacity of the system can be written as,

$$
C=\sum_{k=1}^{K} B \log _{2}\left(1+\frac{\lambda_{k}^{2} p_{k}}{\sigma^{2}}\right)
$$

\section{Power consumption model}

The total power consumption is defined as,

$$
p_{\text {sum }}=\frac{p_{\text {out }}}{\alpha}+N p_{c}+K p_{r} .
$$

Where, the first part is the power amplifier consumption, it is related to the transmitter output power $p_{\text {out }}$ and amplifier efficiency $\alpha$. The latter two parts of (9) are the power consumption of the RF chain. $p_{c}$ and $p_{r}$ are the power consumption at the transmitter and the receiver respectively, the output power is determined by 


$$
p_{\text {out }}=\sum_{k=1}^{K} p_{k}
$$

\section{Definition of Energy Efficiency and Proof of Its Convexity}

Energy efficiency is defined as the total capacity of the system divided by the total power consumption, which can be expressed as,

$$
U=\frac{C}{p_{\text {sum }}}=\frac{\sum_{k=1}^{K} B \log _{2}\left(1+\frac{\lambda_{k}^{2} p_{k}}{\sigma^{2}}\right)}{\frac{\sum_{k=1}^{K} p_{k}}{\alpha}+N p_{c}+K p_{r}} .
$$

\subsection{Proof of convexity of energy efficiency}

Based on the energy efficiency optimization model established in this chapter, with the increase of the user's power, the system energy efficiency increases first and then decreases. The energy efficiency is the convex function of the user's power. That is there is an optimal user power distribution can make the maximum energy efficiency.

Proof: In order to analyze the relationship between the power allocated by the user and the energy efficiency of the system, the energy efficiency function is written as a function of $p_{k}$,

$$
y\left(p_{k}\right)=\frac{\sum_{k=1}^{K} B \log _{2}\left(1+\frac{\lambda_{k}^{2} p_{k}}{\sigma^{2}}\right)}{\frac{\sum_{k=1}^{K} p_{k}}{\alpha}+N p_{c}+K p_{r}} .
$$

In order to judge the relationship between the energy efficiency function and the user power in (12), we can first find the partial derivative of the function with the respect to $p_{k}$, determine the nature of its derivative function. The derivative function after derivative is (13),

$$
\frac{\partial y\left(p_{k}\right)}{\partial p_{k}}=\frac{B \frac{\lambda_{k}^{2}}{\ln 2\left(\sigma^{2}+\lambda_{k}^{2} p_{k}\right)}\left(\frac{\sum_{k=1}^{K} p_{k}}{\alpha}+N p_{c}+K p_{r}\right)-\frac{1}{\alpha} \sum_{k=1}^{K} B \log _{2}\left(1+\frac{\lambda_{k}^{2} p_{k}}{\sigma^{2}}\right) .}{\left(\frac{\sum_{k=1}^{K} p_{k}}{\alpha}+N p_{c}+K p_{r}\right)}
$$

In order to determine the positive or negative properties of (13), the denominator is definitely greater than zero, only need to judge the positive or negative of the molecules. Because the positive or negative of the molecule is not easy to judge, the molecule can be expressed as the (14).

$$
\begin{aligned}
g\left(p_{k}\right)= & B \frac{\lambda_{k}^{2}}{\ln 2\left(\sigma^{2}+\lambda_{k}^{2} p_{k}\right)}\left(\frac{\sum_{k=1}^{K} p_{k}}{\alpha}+N p_{c}+K p_{r}\right) . \\
& -\frac{1}{\alpha} \sum_{k=1}^{K} B \log _{2}\left(1+\frac{\lambda_{k}^{2} p_{k}}{\sigma^{2}}\right)
\end{aligned}
$$

The partial derivative of $g\left(p_{k}\right)$ with the respect to $p_{k}$ is the (15).

$$
\begin{aligned}
\frac{\partial g\left(p_{k}\right)}{\partial p_{k}}= & \frac{-B \lambda_{k}^{4}}{\ln 2\left(\sigma^{2}+\lambda_{k}^{2} p_{k}\right)^{2}}\left(\frac{\sum_{k=1}^{K} p_{k}}{\alpha}+N p_{c}+K p_{r}\right) \\
& +\frac{B \lambda_{k}^{2}}{\ln 2\left(\sigma^{2}+\lambda_{k}^{2} p_{k}\right) \alpha}-\frac{B}{\alpha \ln 2} \frac{1}{1+\frac{\lambda_{k}^{2} p_{k}}{\sigma^{2}}} \frac{\lambda_{k}^{2}}{\sigma^{2}} \\
= & \frac{-B \lambda_{k}^{4}}{\ln 2\left(\sigma^{2}+\lambda_{k}^{2} p_{k}\right)^{2}}\left(\frac{\sum_{k=1}^{K} p_{k}}{\alpha}+N p_{c}+K p_{r}\right)<0
\end{aligned}
$$

(15) is a number less than zero, so $g\left(p_{k}\right)$ is $\mathrm{a}$ decreasing function with respect to $p_{k}$, because

$$
\left.g\left(p_{k}\right)\right|_{p_{k}=0}=B \frac{\lambda_{k}^{2}}{\sigma^{2}}\left(N p_{c}+K p_{r}\right)>0 .
$$

$$
\left.g\left(p_{k}\right)\right|_{p_{k}=+\infty}=\frac{B}{\alpha}\left(\frac{K}{\ln 2}-\lim _{p_{k} \rightarrow \infty} \log 2\left(1+\frac{\lambda_{k}^{2} p_{k}}{\sigma^{2}}\right)\right)<0 .
$$

Since the first part of (17) is a constant and the latter part is infinite when $p_{k}$ tends to infinity, so (17) is less than zero. That is, with the increase of $p_{k}$, the value of $\partial y\left(p_{k}\right) / \partial p_{k}$ is greater than zero and then less than zero. In other words $y\left(p_{k}\right)$ monotonically increasing first and then monotonically decreasing. So there is an optimal $p_{k}$ to make the efficiency function obtain the maximum value.

In summary, with the increase of user power, the system energy efficiency increases first and then decreases, that is, there is an optimal user power to make the efficiency obtain the maximum value. Therefore, through the power distribution algorithm, we can find the optimal value of the energy efficiency function.

\section{The iterative power allocation algorithm}


The energy efficiency function is the convex function of $p_{k}$. Therefore, the optimal value of user power can be obtained by solving the derivative of the function $U$ with the respect to $p_{k}$, then letting the derivative function equal to zero, we can obtain,

$$
p_{k}=\left\{\begin{array}{cl}
\frac{\alpha B}{U \ln 2}-\frac{\sigma^{2}}{\lambda_{k}^{2}} & \text { if } \frac{\alpha B \lambda_{k}^{2}}{\sigma^{2} \ln 2}>U \\
0 & \text { else }
\end{array}\right.
$$

(18) has gotten the expression of the user power optimal value, but the expression of $U$ implies $p_{k}$, we can not get the optimal value of the user power directly. Therefore, an iterative power allocation algorithm is proposed to obtain the optimal value. Define:

$$
f\left(p_{k}\right)=\sum_{k=1}^{K} B \log _{2}\left(1+\frac{\lambda_{k}^{2} p_{k}}{\sigma^{2}}\right)-U\left(\frac{\sum_{k=1}^{K} p_{k}}{\alpha}+N p_{c}+K p_{r}\right) \text {. }
$$

(18) and (19) can get the energy efficiency of the system by updating and iterating constantly. When the maximum energy efficiency is obtained, (19) should be zero, so the iteration continues until (19) is sufficiently small to meet the convergence requirement. This power allocation algorithm is summarized in Table 1.

\section{Table 1. An iterative power allocation algorithm}

\begin{tabular}{|l|l|}
\hline $\begin{array}{l}\text { Step } \\
1:\end{array}$ & $\begin{array}{l}\text { assuming that the average allocation of power } \\
\text { that is } p_{k}^{1}=p_{\text {out }} / K, \text { take } p_{k}^{1} \text { and } U^{1}=0 \text { into } \\
\text { (19) to get } f^{1} .\end{array}$ \\
\hline $\begin{array}{l}\text { Step } \\
2:\end{array}$ & $\begin{array}{l}\text { take } U^{2}=f^{1} \text { into (18) to get } p_{k}^{2}, \text { then take } p_{k}^{2} \\
\text { and } U^{2} \text { into the }(19) \text { to calculate the energy } \\
\text { efficiency } f^{2} .\end{array}$ \\
\hline $\begin{array}{l}\text { Step } \\
\text { n: }\end{array}$ & $\begin{array}{l}\text { take } p_{k}^{n} \text { and } U^{n} \text { into the (19) to calculate } f^{n} \text { at } \\
\text { the same time, if } f^{n}<0.001, \text { then the iteration } \\
\text { ends. Otherwise continue to step n+1. }\end{array}$ \\
\hline
\end{tabular}

\section{Simulation results}

The following is the simulation results to verify the effectiveness of the algorithm. Assuming that the base station bandwidth is $10 \mathrm{kHz}$, the power consumption of each RF chain at the transmitter and receiver is $100 \mathrm{~mW}$. The noise power is $-114 \mathrm{dBW} / \mathrm{MHz}$ and the power amplifier efficiency is 0.5 . Define the equal power allocation algorithm is that the transmitted power is allocated equally for each user,

$$
p_{k}=\frac{p_{\text {out }}}{K} .
$$

Figure 3 shows the relationship between the power consumption and the number of antennas. From the figure 3 , we can see that, with the increase of the transmit antenna number, the total power consumption of the system is constantly increasing, and the more users at the receiver, the greater the power consumption. This is because under this paper's power consumption model, the total power consumption is a linear relationship with the number of transmitting antennas and users

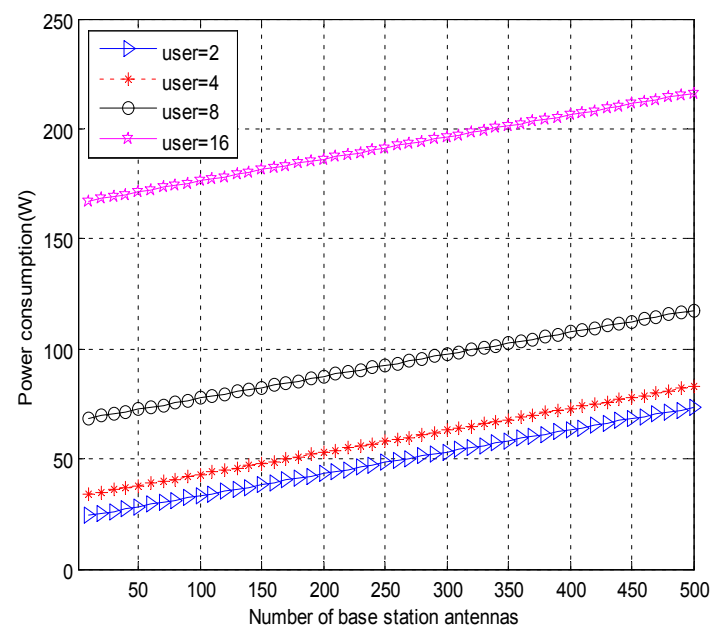

Figure 3. Relationship between power consumption and transmitting antennas number

Figure 4 is an energy efficiency comparison between the iterative power allocation algorithm and the equal power allocation algorithm when the number of base station antennas is 128 . It can be seen that the energy efficiency of the iterative power allocation algorithm is higher than the equal allocation power. Because the user's power in iterative power allocation algorithm is optimal, it is independent with the output power of base station, and it can be approximately a straight line. However when the user allocates the output power equally, the energy efficiency increases first and then decrease, that is because the power consumption increases with the increase of output power, but when the output power is less than $32 \mathrm{dBm}$, the system capacity increases more than the power consumption, the energy efficiency increases. When the output power is greater than $32 \mathrm{dBm}$, the system capacity increases less than the power consumption, so energy efficiency reduces. At the same time we can see 
that in the same algorithm the more users, the greater the energy efficiency.

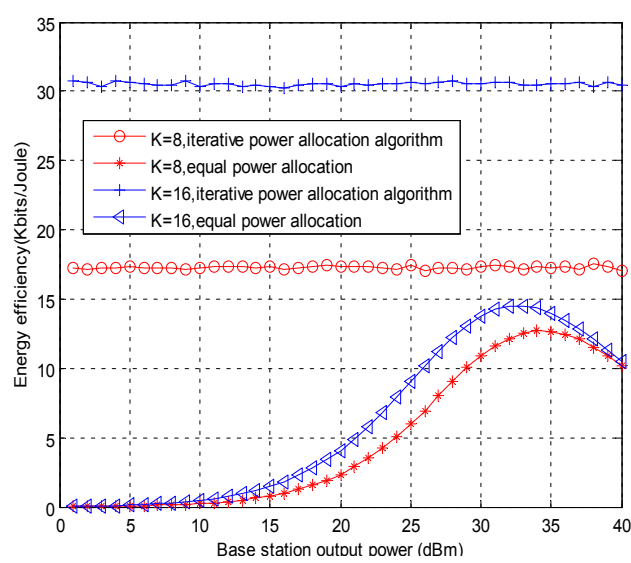

Figure 4. Energy efficiency comparison between iterative power allocation algorithm and equal power allocation

Figure 5 shows the relationship between the number of base station antennas and the energy efficiency. It can be seen that with the increase of base station antenna number, the energy efficiency increases first and then decrease, in other words, there exists an optimal number of base station antennas to maximize energy efficiency. This is because when the number of base station antennas is small, with the increase of the transmitting antenna number, the RF chain consumes less power and the system capacity increases more quickly, so the energy efficiency increases. However, when the number of base station antennas is large, the loss of the RF chain becomes large and the capacity increases slow relatively, so the energy efficiency reduces. And the more number of users, the greater energy efficiency of the system.

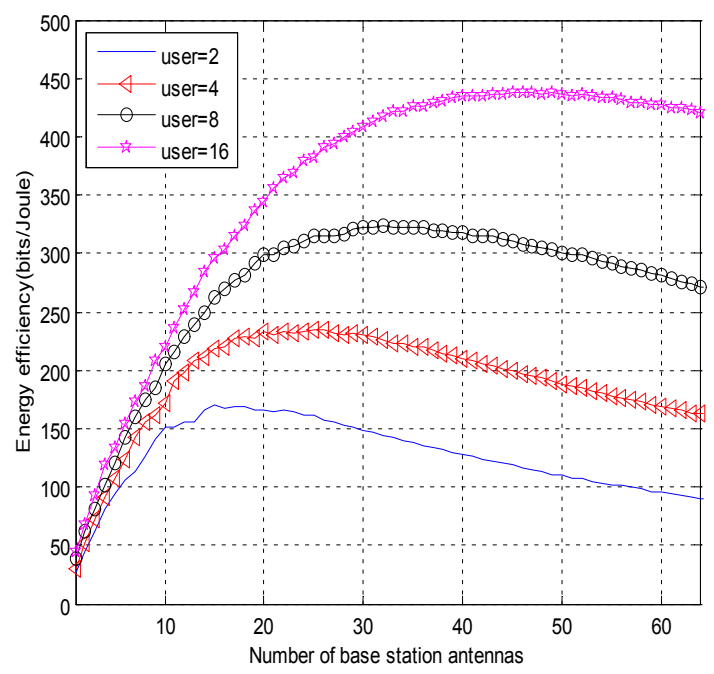

Figure 5. Energy efficiency of the iterative power allocation algorithm

\section{Summary}

In this paper, an iterative power allocation algorithm is proposed to improve the energy efficiency of Massive MIMO systems. Firstly, a new power model is founded, which takes the users' power and circuit power into account. Based on this model, we deduce the expression of energy efficiency and an iterative power allocation algorithm is proposed by using convex optimization to obtain the optimal value of user power. Then the influence of the algorithm on the total power consumption of the system is analyzed. The power efficiency of the proposed power algorithm and average power allocation is compared. The simulation results show that the proposed iterative power allocation algorithm can significantly improve the energy efficiency of the system.

\section{References}

[1] Lee, B.M, Choi, J.H, Bang, J.H.: An energy efficient antenna selection for large scale green MIMO systems[C]. IEEE International Symposium on Circuits and Systems. IEEE, 2013:950-953.

[2] Ngo, H.Q, Larsson, E.G, Marzetta, T.L.: Energy and Spectral Efficiency of Very Large Multiuser MIMO Systems [J]. IEEE Transactions on Communications, 2013, 61 ( 4 ): 1436-1449.

[3] Hamdi, R. and Ajib, W.: Joint Optimal Number of RF Chains and Power Allocation for Downlink Massive MIMO Systems. Vehicular Technology Conference (VTC Fall), 2015 IEEE 82nd, Boston, MA, 2015, pp.1-5.

[4] Wang, A. Y, Chao, S, Sodini, C.G, and Chandrakasan, A.P.: Energy efficient modulation and MAC for asymmetric RF microsensor system. In Proc. 2001 Int. Symp. Low Power Electronics and Design, pp. 106-111.

[5] Pei, Y, Pham, T.H, Liang, Y.C.: How many RF chains are optimal for large-scale MIMO systems when circuit power is considered?[C] Global Communications Conference. IEEE, 2013:3868-3873.

[6] Cui, S, Goldsmith, A. and Bahai, A.: Energy-efficiency of MIMO and cooperative MIMO techniques in sensor networks. In IEEE J. Sel. Areas Commun., vol. 22, no. 6, Aug. 2004, pp. 1089-1098.

[7] Kim, E.P. And Shanbhag, N.R.: An energy-efficient multiple-input multiple-output (MIMO) detector architecture. In 2011 IEEE Workshop on Signal Processing Systems.

[8] Li, G.Y, Xu, Z, Xiong, C.: Energy-efficient wireless communications: tutorial, survey, and open issues[J]. IEEE Wireless Communications, 2011, 18(6): 28-35.

[9] Yuming Miao,Xiaoyan Huang,Supeng Leng.Optimal Energy Efficiency Allocation in OFDMA System[J].Journal of Electronics \& Information Technology,2014,36(7)(in Chinese).

[10] Feng, W, Chen, Y, Ge, N, Lu, J.: Optimal Energy-Efficient Power Allocation for Distributed Antenna Systems with Imperfect CSI. In IEEE Transactions on Vehicular Technology, vol.PP, no.99, pp.1-1.

[11] Miao, G.: Energy-Efficient Uplink Multi-User MIMO. In IEEE Transactions on Wireless Communications, vol. 12, no. 5, pp. 2302-2313, May 2013. 